\title{
Activity Routine Discovery in Stroke Rehabilitation Patients without Data Annotation
}

\author{
Julia Seiter \\ Wearable Computing Lab. \\ ETH Zurich \\ Zurich, Switzerland \\ seiter@ife.ee.ethz.ch
}

\author{
Adrian Derungs \\ ACTLab \\ University of Passau \\ Passau, Germany
}

\author{
Corina Schuster-Amft \\ Research Department \\ Reha Rheinfelden \\ Rheinfelden, Switzerland
}

\author{
Oliver Amft \\ ACTLab \\ University of Passau \\ Passau, Germany
}

\author{
Gerhard Tröster \\ Wearable Computing Lab. \\ ETH Zurich \\ Zurich, Switzerland
}

\begin{abstract}
In this work, we investigated whether activity routines of stroke rehabilitation patients can be discovered from bodyworn motion sensor data and without data annotation using topic modeling. Information about the activity routines performed by stroke patients during daily life could add valuable information to personal therapy goals. As topic model input, we used a set of activity primitives derived from upper and lower extremity motion sensor data. We monitored three stroke patients during their daily life in a day care center for 8 days each within 3 weeks. We achieved up to $88 \%$ accuracy for activity routine discovery for subject-dependent evaluations. Our discovery approach seems suitable for activity routine discovery in rehabilitation patients.
\end{abstract}

\section{Categories and Subject Descriptors}

H.4 [Information Systems Applications]: Miscellaneous

\section{General Terms}

Design, Experimentation, Human Factors, Measurement

\section{Keywords}

Topic model, stroke patients, unsupervised, daily routines

\section{INTRODUCTION}

Stroke is considered to be the most leading cause of disability in the world [8]. To assess the physical impairment of stroke patients therapists use motor function tests, as the FuglMeyer and the Chedoke-McMaster Stroke Assessment [4, 5]. However, these clinical assessments can only be applied during therapy and do not provide information on patients' activities and routines in daily life. Several approaches exist to implement objective activity measurements in daily life $[9,6,11]$. Patel et al. estimated the total Functional Ability

Permission to make digital or hard copies of all or part of this work for personal or classroom use is granted without fee provided that copies are not made or distributed for profit or commercial advantage and that copies bear this notice and the full citation on the first page. To copy otherwise, to republish, to post on servers or to redistribute to lists, requires prior specific permission and/or a fee. REHAB 2014, May 20-23, Oldenburg, Germany Copyright (C) 2014 ICST 978-1-63190-011-2 DOI 10.4108/icst.pervasivehealth.2014.255275
Scale score of patients from acceleration data [9]. Uswatte et al. used accelerometers at the wrists to infer the activity of the impaired arm [11]. While these approaches provide quantitative scores and activity measures, they do not yield insight in the type of activities and daily routines that patients performed. Nevertheless, monitoring patients' behavior and activity routines outside therapy could add valuable information on describing patient lifestyle and thus defining individual therapy goals.

Activity discovery has been proposed to identify daily routines, without previously trained classifiers $[1,3]$. However, activity routine discovery needs to deal with variations in routine performance from day to day and between persons. A commonly considered approach to activity routine discovery is to compose routines from activity primitives. Activity primitives have a fine temporal granularity and can be recognized from on-body sensor data. Huynh et al. applied topic models to discover activity routines, such as lunch and office work from activity primitives [7]. They used activity primitives including desk activity and having lunch, which were classified using a previously trained Naïve Bayes model. These activity primitives were subsequently considered as input for the topic model. Since the activity primitives required a trained classifier, annotations of actual activity performances would be needed during the classifier training. In contrast, we investigate whether the activity routines of rehabilitation patients could be discovered from activity primitives without the need of trained classifiers. Our approach is based on person-independent body posture and activity features measured at the extremities.

In this work, we investigate a topic model-based activity routine discovery in rehabilitation patients using wearable motion sensors. Our approach does not require annotations for activity primitives and routines. In particular, the contributions of this paper are the following: (1) we show that activity routines can be discovered from a set of rule-based, person-independent activity primitives that do not require trained classifiers and activity annotations. (2) We analyzed the influence of the key topic model parameters document size, number of activity topics, and the hyperparameter $\alpha$, on the activity routine discovery performance. (3) We 
evaluated our approach with three stroke patients who were recorded in a day care center using wearable motion sensors during 8 days within 3 weeks.

\section{METHODOLOGY}

For activity routine discovery, we used a layered approach as illustrated in Figure 1a. Activity primitives were derived from sensor data according to a set of rules. Subsequently, the activity primitives were used as input for topic model based activity routine discovery. Activity topic activations (probabilities) were then mapped to distinct activity routines. The study recording process and the different layers of the discovery approach are detailed in the following.

\subsection{Study Recordings}

In our monitoring study, we included three male stroke patients, aged $47-57$ years. The patients regularly visited the day care center of the Reha Rheinfelden rehabilitation center in Switzerland. Patients suffered from hemispheric stroke resulting in either left or right upper and lower extremity activity impairment. Two of three patients primarily used a wheelchair but were capable of short distance walking. Patients arrived in the day care center in the morning, followed their daily therapy schedule including lunch and resting phases and went home in the evening. In the morning, 6 Shimmer3 motion sensors were attached to each wrist, upper arm and upper leg as illustrated in Figure 1b and logged acceleration, gyroscope and magnetometer data at $50 \mathrm{~Hz}$. Sensors were carried during the whole day (except for temporary removal during water therapy) and detached before patients left the day care center in the evening. The study was approved by the local cantonal Ethics committee.

For recording days, activity routine annotations were extracted from the individual daily therapy schedule by assigning each therapy to one of the activity routines. Activity routines included cognitive training (covering training exercises on a computer or working sheet) socialising (active

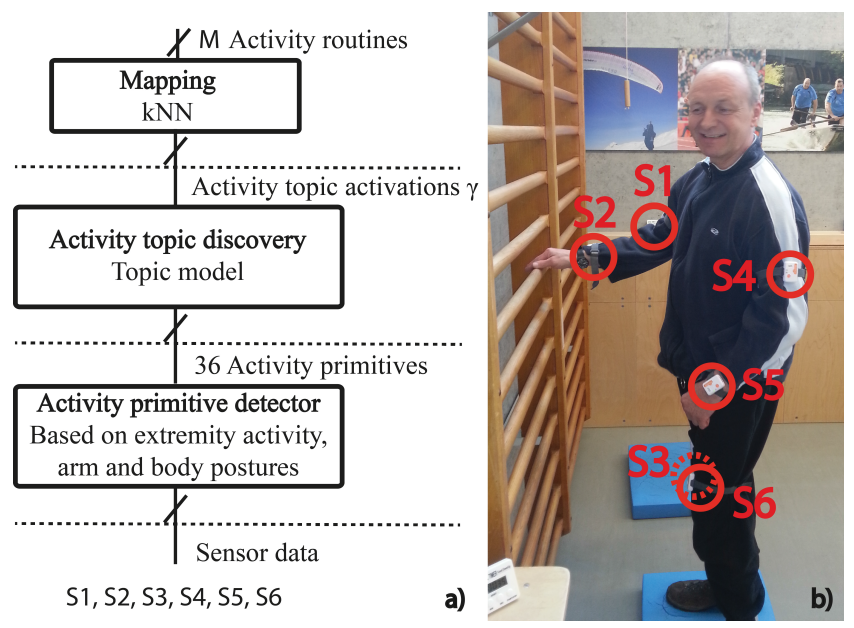

Figure 1: a) Overview of activity routine discovery approach: Activity primitives are detected from sensor data $S 1-S 6$. Subsequently activity topics are discovered from activity primitives followed by $\mathrm{kNN}$ based mapping of activity topics to activity routines, b) sensor setup with affected arm left.
Table 2: Number of activity routine repetitions recorded for each patient in 8 days within 3 weeks.

\begin{tabular}{l|l|l|l|l} 
& Patient 1 & Patient 2 & Patient 3 & Total \\
\hline \hline Cognitive training & 3 & 1 & 5 & 9 \\
\hline Socialising & 34 & 33 & 35 & 102 \\
\hline Motor training & 13 & 20 & 14 & 47 \\
\hline Medical fitness & 2 & 8 & 7 & 17 \\
\hline Rest/sleep & 1 & 6 & 4 & 11
\end{tabular}

interaction with other people) motor training (therapies that involve physical motor function training exercises) medical fitness (intense physical training exercises) and rest/sleeping phases. Rest/sleeping phases were not specified in the therapy schedule but performed during breaks in the day care center. Thus, time and duration of rest/sleeping were added to the therapy schedule (hand written) by therapists and the study examinators. In total, we collected 137 hours of data, of which 100 hours were annotated. For each patient 8 days within 3 weeks were recorded. Depending on patients' personal therapy schedule, only a subset of activity routines might be performed regularly. Table 2 shows the routines and number of repetitions recorded for each patient.

\subsection{Activity Primitive Detector}

In total, we derived 36 activity primitives from upper arm and lower arms and thigh-worn sensors. Activity primitives were described by binary decisions on particular arm and body postures as well as arm and leg movement as detailed in Table 1. We considered arm and leg activity including affected an non-affected extremities to be indicators for the physical activity of patients and thus relevant for activity routine discovery. We further distinguished between activity in the low frequency band $(0.2-2.5 \mathrm{~Hz})$ and outside the low frequency band. We also assumed body postures like sitting and standing and arm postures such as the angle between upper and lower arm, wrist orientation and orientation of the lower arm towards the horizontal plane to be informative for activity routine discovery in rehabilitation scenarios.

\subsection{Activity Topic Discovery and Mapping}

We applied the Latent Dirichlet Allocation topic model (LDA) for activity topic discovery as suggested by [7]. In activity routine discovery the topic model is used to discover $K$ hidden activity topics in a corpus of segments. Each segment covers histograms over activity primitive sequences of a time segment $D S$ of a day. LDA assumes that for each segment $s$ there is an activity topic distributions $\theta_{s}$ which is derived from a Dirichlet distribution $\operatorname{Dir}(\alpha)$ with the hyperparameter $\alpha$. While a high $\alpha$ value should favor all activity topics in a segment equally, small $\alpha$ would privilege one particular activity topic for one segment. When applying LDA to a corpus of segments a $K$-dimensional activity topic activation vector $\gamma_{s}$ is inferred from the activity primitive histogram of each segment $s$ based on the estimated distribution $\theta_{s}$. The normalized $\gamma_{s}$ describes the estimated occurrence ratio of each activity topic in a segment $s$. More detailed information on LDA can be found in [2]. As suggested by [7] the number of activity topics $K$ in the data could be higher than the number of activity routines $M$. In order to assess and compare discovery accuracy across patients we applied a $\mathrm{kNN}$ classifier for mapping activity topics to activity routines using the topic activation vector $\gamma_{s}$ as feature vector. 
Table 1: Activity primitives (1)-(36) detected at the low-level activity primitive detector: The table shows feature window sizes/steps, thresholds of binary features $F_{S i}$ inferred from the 3-axis acceleration signal $a c c_{x y z}$ and quaternions $\left(q_{S i}, q_{S j}\right)$ for sensors $S i, j \mid \in\{S 1, S 2 . ., S 6\}$ as well as detection logic for each activity primitive.

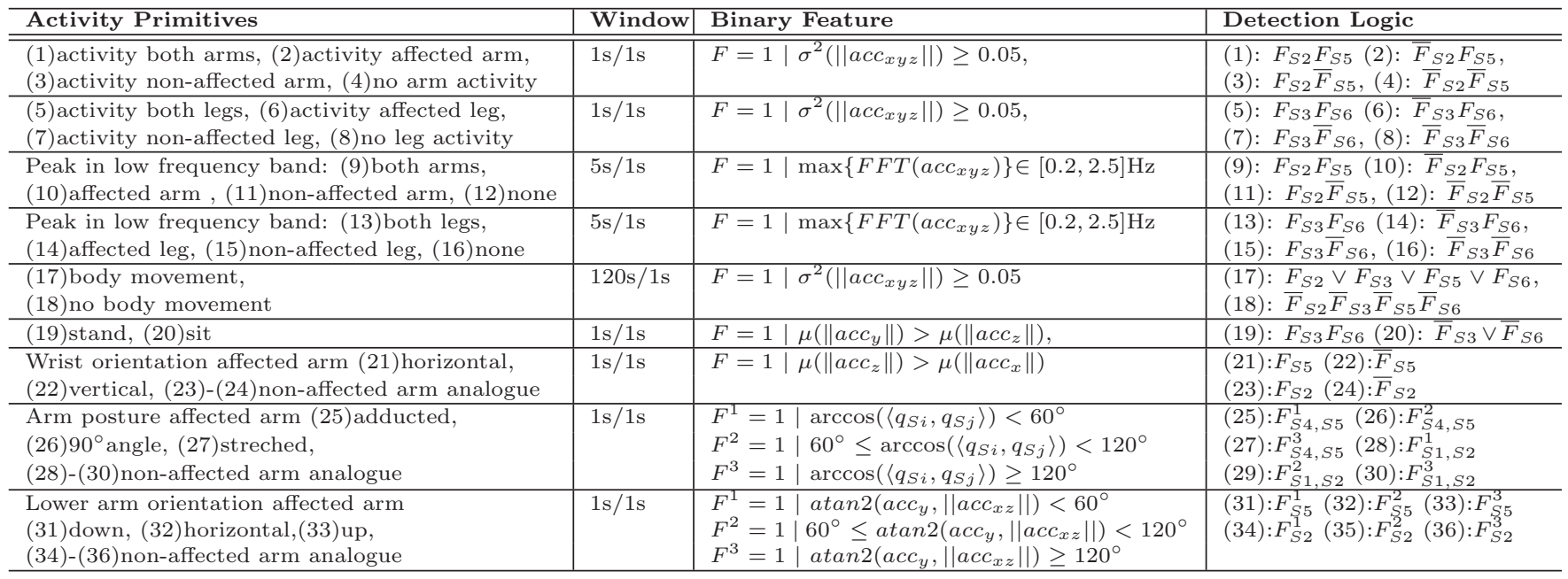

\subsection{Implementation}

To evaluate the topic model we used the LDA implementation of [2], which includes an iterative optimization for topic model parameters $\alpha, \theta$ regarding model likelihood. The initial hyperparameter $\alpha$ was set to $\alpha=0.01$ as suggested by [7]. Activity primitive segments were formed of segment size $D S$ with a segment step $D W=0.1 \cdot D S$. We applied the Borda Count ranking method to the topic activations $\gamma_{s}$ of all segments $s$ covering the same $D W$ time slot. We investigated subject-dependent leave-one-day-out cross-validation and subject-adapted leave-one-day-out cross-validation. The evaluations involved topic model and $\mathrm{kNN}$ model estimation on all patients' data except for the left-out day. For the evaluation analysis we only evaluated activity routines counting at least 3 repetitions in the dataset resulting in 3 , 4,5 and 5 activity routines for patient 1,2, 3 and subjectadapted analysis (Table 2). For each topic model estimation we performed 3 iterations and chose the one with the highest likelihood. As evaluation measure we used the averaged class-specific accuracy of activity routine predictions on activity routine annotations. Because of random topic model internal parameter initialization we investigated mean and standard deviation across 5 independent topic model runs. We further investigated the influence of the hyperparameter alpha on the discovery performance. Thus, we evaluated fixed alpha values beyond the suggested default setting $50 / K[10]$.

\section{RESULTS}

\subsection{Activity Routine Discovery}

Figure 2 shows the averaged class-specific accuracies for the subject-dependent and subject-adapted evaluation for different segment sizes $D S$ and number of activity topics $K$. Using our layered discovery approach the activity routines of rehabilitation patients were discovered with up to $88 \%$ accuracy for patients 1 and 2 and about $75 \%$ for patient 3 . The evaluation included activity routines as specified in Section 2.4. With increasing number of topics, accuracies even increased for all patients. For all patients, the highest accuracies could be achieved at a segment size of $D S=20$ min. At comparable parameters for subject-dependent mod- els (segment size of $D S=20 \mathrm{~min}$ and $K=2 M$ ) we achieved $78 \%$ accuracy for patient $1(K=6)$ and $3(K=10)$ and $88 \%$ for patient $2(K=8)$. Patient 2 was not using a wheelchair and the recordings showed larger variability in activity primitives across the activity routines, which could explain the overall higher accuracy obtained for this patient. We even yielded $71 \%$ accuracy for subject-adapted evaluation including all activity routine data (also routines with less than 3 repetitions per patient, Table 2 ).

Figure $3 \mathrm{~b}$ shows discovery performances across different $\alpha$ values exemplary for patient 3 . The parameter rarely has an influence on the accuracy as variations are small and within the accuracy's standard deviation (Figure 2). This trend is similar for all 3 patients. Thus, routine topic activations $\gamma$ inferred from activity primitives seem to be discriminative,

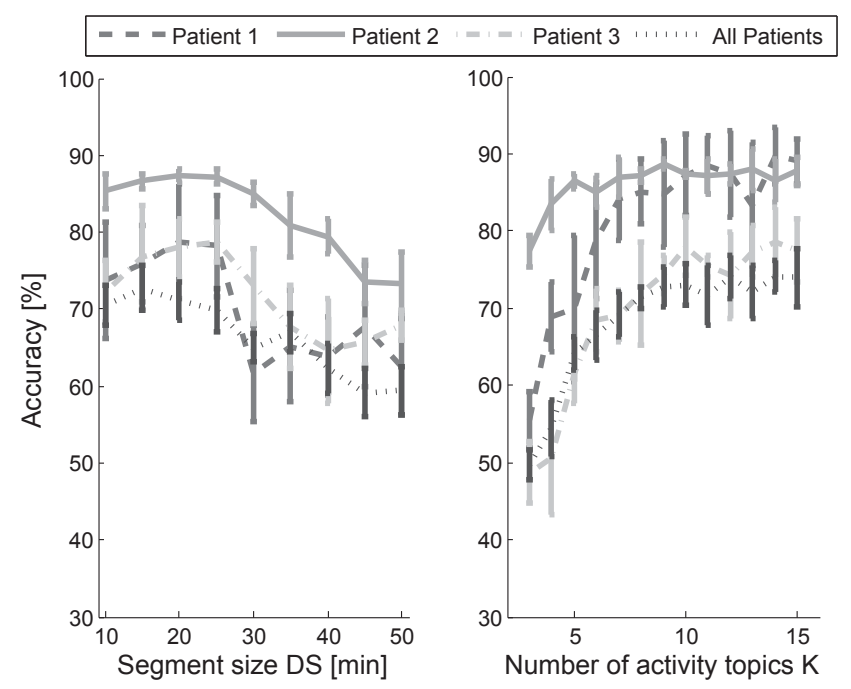

Figure 2: Averaged class specific accuracies and standard deviation for varying segment size $D S$ and number of activity topics $K$ for subject-dependent (patients 1,2 and 3) and subject-adapted (all patients) evaluation. 


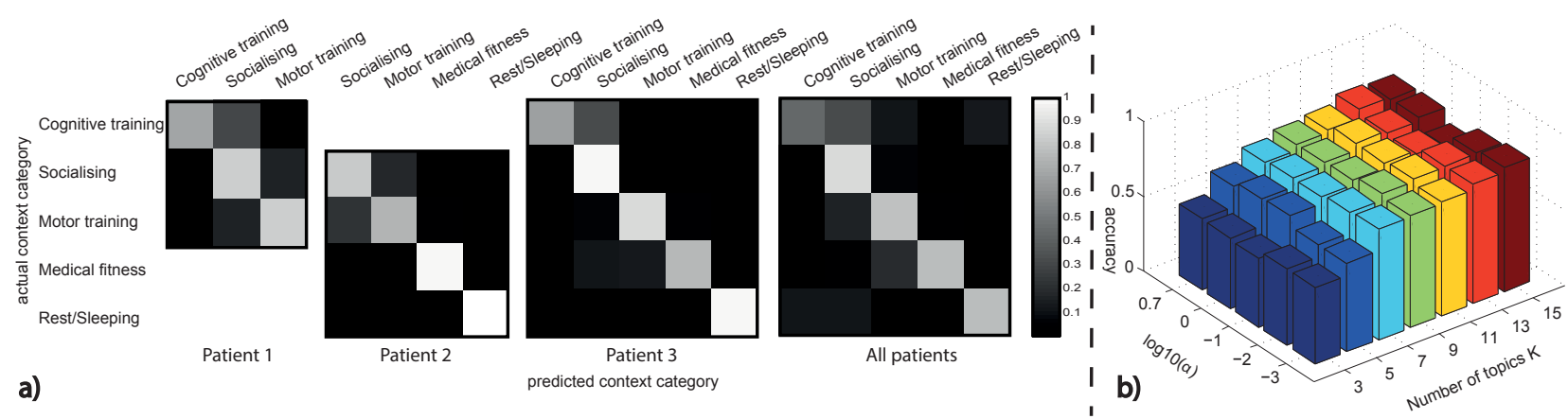

Figure 3: a) Confusion matrices for subject-dependent (patients 1, 2, 3) and subject-adapted (all patients) evaluation showing patient specific activity routine classes. Activity routines are clearly separable from activity primitives based on body posture and extremity activity, b) averaged class specific accuracies for different topic model hyperparameters $\alpha$ and number of topics $K$. The hyperparameter does not influence the discovery accuracy of the approach as variations are small and within the standard deviation of $5 \%$.

independent of $\alpha$. Having discriminative routine topic activations the kNN (used for mapping and performance evaluation) yields high accuracies. However, when targeting activity routine discovery favored and thus clear activated activity topics (small $\alpha$ values) per segment could make routine topic and activity routine mapping more evident. The investigate this hypothesis an alternative to $\mathrm{kNN}$ for performance evaluation should be analyzed in future work.

Fig. 3a depicts the confusion matrices for subject-dependent and -adapted evaluations. Using the layered discovery approach all activity routine patterns were clearly separable for each patient. Furthermore, few confusions for the subjectadapted analysis suggest activity routine patterns to be similar across patients. The $6 \%$ decrease in averaged accuracy compared to subject-dependent analysis (Figure 2) results from increasing confusions for cognitive training and socialising. Reasons might be highly patient dependent execution of the activity routine cognitive training and few activity routine repetitions (total $=9$, Table 2 ). Overall high accuracies show that it is possible to discover activity routines of stroke patients from sensor data using topic modeling. The topic model discovered meaningful activity routine patterns from activity primitive derived from upper and lower extremity activity and body and arm postures.

\section{CONCLUSION}

In this paper, we investigated activity routine discovery of rehabilitation patients from sensor data using topic modeling. We achieved accuracies between $78 \%$ and $88 \%$ for all subject-dependent evaluations when considering activity routines including socialising, cognitive training, medical fitness, motor training and rest/sleeping phases. These results indicate that our approach can be suitable for the discovery of activity routines that rehabilitation patients perform during a day in the day care center. The topic model parameter invstigation showed that with increasing number of activity topics, accuracy increased for all subject. While the optimal accuracy was found for a segment size of $D S=20 \mathrm{~min}$ the hyperparameter $\alpha$ did not influence accuracy. High discovery accuracies suggests that the activity routines show characteristic patterns regarding the derived set of activity primitives which was based on arm and leg movement as well as arm and body postures for both, affected and nonaffected body side. Thus, activity routine disocvery does not require complex activity primitives detected from trained classifiers. Furthermore, the subject-adapted investigation showed, that activity routine patterns seem to be highly similiar across all three stroke patients. In future work, we plan to validate our approach by extending the study at the day care center and expand the evaluations to a higher number of patients.

\section{ACKNOWLEDGMENTS}

This work was supported by the EU Marie Curie Network iCareNet, grant number 264738. We are grateful to the participating patients and therapists from the day care center.

\section{REFERENCES}

[1] T. S. Barger et al. Health-status monitoring through analysis of behavioral patterns. Systems, Man and Cybernetics, Part A: Systems and Humans, IEEE Transactions on, 35(1):22-27, 2005.

[2] D. Blei et al. Latent dirichlet allocation. JMLR, 2003.

[3] K. Farrahi and D. Gatica-Perez. Discovering routines from large-scale human locations using probabilistic topic models. ACM Trans. Intell. Syst. Technol., 2011.

[4] A. R. Fugl-Meyer et al. The post-stroke hemiplegic patient. 1. a method for evaluation of physical performance. SCAND J REHABIL MED, 1974.

[5] C. Gowland et al. Measuring physical impairment and disability with the chedoke-mcmaster stroke assessment. Stroke, 24:58-63, 1993.

[6] E. Haeuber et al. Accelerometer monitoring of home-and community-based ambulatory activity after stroke. ARCH PHYS MED REHAB, 2004.

[7] T. Huynh et al. Discovery of activity patterns using topic models. In UbiComp, 2008.

[8] W. H. Organization. The world health report 2003: shaping the future. World Health Organization, 2003.

[9] S. Patel et al. A novel approach to monitor rehabilitation outcomes in stroke survivors using wearable technology. P IEEE, 2010.

[10] M. Steyvers and T. Griffiths. Probabilistic topic models. Handbook of latent semantic analysis, 2007.

[11] G. Uswatte et al. Ambulatory monitoring of arm movement using accelerometry: an objective measure of upper-extremity rehabilitation in persons with chronic stroke. ARCH PHYS MED REHAB, 2005. 